\title{
Pleural Biopsies: Just Freeze It?
}

\author{
Sabina Berezowska ${ }^{a}$ Christophe von Garnier ${ }^{b}$

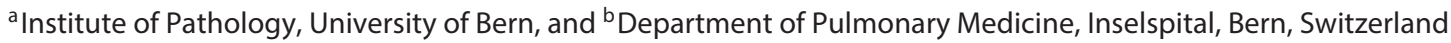

An unclear pleural effusion is a frequently encountered problem and thoracoscopic pleural biopsies may be necessary to establish the diagnosis. Also, pleurodesis most commonly with talc - is part of the state-of-the-art management of malignant pleural effusions in selected patients and should ideally be performed concomitantly with the diagnostic intervention. In the current issue of Respiration, Karpathiou et al. [1] address the clinically relevant question how accurately frozen sections of thoracoscopic pleural biopsies establish the diagnosis of malignancy versus the absence thereof. Following the evaluation of 156 consecutive specimens, a diagnosis was rendered in 149 (95.5\%) cases on frozen section examination ( 97 malignant, 52 benign), and 7 cases (4.5\%) were inconclusive. When compared with the final diagnosis after conventional formalin fixation, paraffin embedding, hematoxylin and eosin staining, and the application of additional auxiliary techniques if needed, 144 out of 156 (92.3\%) diagnoses were concordant, but 5 (3.2\%) were discrepant, with 1 false positive and 4 false negative calls. The authors derive a sensitivity of $96.26 \%$, a specificity of $97.87 \%$, a negative predictive value of $92 \%$, and a positive predictive value of $99.04 \%$. Therefore, the most salient finding from this study is that diagnosis of malignancy on frozen sections is feasible, reliable, and may be followed by immediate pleurodesis during the same procedure.

The accuracy of pleural frozen section examination has been assessed in only few reports so far, and the data

\section{KARGER}

(C) 2017 S. Karger AG, Basel

E-Mail karger@karger.com

www.karger.com/res reported by Karpathiou et al. [1] are very encouraging. The diagnostic accuracy of frozen sections critically depends on the organ site, with the highest number of diagnoses deferred until final histology reported for specimens from the lung/pleural/mediastinal region (13\%) [2]. The type of malignancy is also of importance, as exemplified by the difficulties that the discrimination of malignant pleural mesothelioma (MPM) from reactive changes may pose to capture invasive growth, even on final histological examination, and adequate sampling may be of utmost importance in this context [3]. Accordingly, in a series of 20 medical thoracoscopy specimens, only 2 out of 6 MPMs were called malignant during intraoperative examination [4]. In the present study, the rate of false negative and deferred diagnoses was overall significantly lower, but also highest $(5 / 31,16.1 \%)$ in the MPM group. The superior accuracy could be due to presumably different thoracoscopic techniques applied and ultimately also specimen dimensions. Although pure biopsy size did not affect diagnostic accuracy of frozen section examination, biopsy depth - important for judging invasive growth - was not examined, and Karpathiou et al. [1] did not specify the method of biopsy acquisition. As semi-rigid techniques employing smaller flexible forceps become more widely available, smaller biopsy size and reduced depth may become a relevant issue affecting diagnostic performance of frozen sections, warranting further studies $[5,6]$.

Prof. Dr. med. C. von Garnier

Universitätsklinik für Pneumologie, Spital Tiefenau

Tiefenaustrasse 112

CH-3004 Bern (Switzerland)

E-Mail christophe.vongarnier@insel.ch 
Also, in subsequent studies it would be desirable to provide the diagnostic criteria for malignancy present in the individual cases assessed and their impact on diagnosis. Was infiltrative growth a sine qua non for diagnosing malignancy on frozen sections? And if not, in how many cases was it present? Loss of BAP1 expression assessed by immunohistochemistry and p16 deletion assessed by fluorescence in situ hybridization have recently proven valuable specific markers confirming malignancy in mesothelial lesions, enabling definite diagnosis, even in cytology specimens $[7,8]$. Staining for cytokeratin can highlight invasive growth, and other immunohistochemical markers help to diagnose carcinoma infiltrates [3]. This may thus have an impact on the necessity of future diagnostic approaches with pleural frozen sections. Ultimately, the added value of pleural frozen sections needs to be placed in the context of the ever-evolving landscape of available auxiliary techniques that may be necessary to reach the final diagnosis.

Although the study by Karpathiou and colleagues is retrospective in nature, with inherent limitations, it sheds light on an important domain and clinically relevant question, providing encouraging data for both clinicians and pathologists.

\section{References}

1 Karpathiou G, Froudarakis M, Forest F, et al: Frozen sections in pleural pathology: a valuable tool. Respiration 2017;94:45-51.

2 da Silva RDP, Souto LRM, Matsushita G de M, Matsushita M de M: Diagnostic accuracy of frozen section tests for surgical diseases. Rev Col Bras Cir 2017;38:149-154.

3 Husain AN, Colby T, Ordonez N, et al: Guidelines for pathologic diagnosis of malignant mesothelioma: 2012 update of the consensus statement from the International Mesothelioma Interest Group. Arch Pathol Lab Med 2013;137:647-667.
4 Fielding D, Hopkins P, Serisier D: Frozen section of pleural biopsies at medical thoracoscopy assists in correctly identifying benign disease. Respirology 2005;10:636-642.

5 Dhooria S, Singh N, Aggarwal AN, Gupta D, Agarwal R: A randomized trial comparing the diagnostic yield of rigid and semirigid thoracoscopy in undiagnosed pleural effusions. Respir Care 2014;59:756-764.

6 Rozman A, Camlek L, Marc-Malovrh M, Triller N, Kern I: Rigid versus semi-rigid thoracoscopy for the diagnosis of pleural disease: a randomized pilot study. Respirology 2013; 18:704-710.
7 Cigognetti M, Lonardi S, Fisogni S, et al: BAP1 (BRCA1-associated protein 1) is a highly specific marker for differentiating mesothelioma from reactive mesothelial proliferations. Mod Pathol 2015;28:1043-1057.

8 Nabeshima K, Matsumoto S, Hamasaki M, et al: Use of p16 FISH for differential diagnosis of mesothelioma in smear preparations. Diagn Cytopathol 2016;44:774-780. 